\title{
A statistical method for stastical appraisal of the power cable conditions based on the TD and PD diagsnostics result
}

\author{
T. Neier ${ }^{1, *}, J$. Knauel $^{1}, M$. Bawart $^{1}, D$. Antipov $^{1}$, and $S . \mathrm{Kim}^{2}$ \\ ${ }^{1}$ BAUR GmbH, Austria \\ ${ }^{2}$ KEPCO, South Korea
}

\begin{abstract}
This study handles one of the key questions of network operators: How can the remaining life time of underground power cables be estimated? The answer to this question is explained by a new method of KEPCO Korea. When combining VLF Tan Delta (TD) and Partial Discharge (PD) diagnostic it is possible to identify and localize weak individual spots along a cable. After weak spots are cleared, the general aging condition of the cable can be evaluated and the Remaining Life Time can be estimated. The implementation of this approach in the KEPCO Distribution Networks is illustrated in a practical case study. A new tool for asset managers is available and it is expected that it will help to further develop the preventive maintenance approach by power utilities all around the world.
\end{abstract}

\section{Introduction}

The importance of a reliable medium voltage cable network is well understood by the cable operators today. When planning the maintenance of these networks DSOs have to manage the conflicting requirements of scarce resources, high standards in terms of service security and the issue of networks that become older and more complex. In addition, the energy transition towards renewable energy sources pushes underground cable networks to their limit.

Very Low Frequency (VLF) Monitored Withstand Diagnostic was found to be a very effective tool for the evaluation of the cable condition. Based on the results a decision on the urgency of partial or sectional replacement can be made [3]. The estimation of the remaining life time of a cable is the challenging key for DSOs in order to manage investments and resources. In Europe it can be assumed that the average costs for the installation of a new cable route is in the range of $100,000 € / \mathrm{km}$. Commonly the life expectancy of a XLPE cable can be assumed with 30 to 40 years. A reliable diagnostic tool allows categorizing an old cable to be still in a healthy condition. Therefore, the cable can remain in service for another 10 years. The resulting extension of the life expectancy a particular cable route can be quantified up to $25 \%$ of the initial investment cost. In case this applies to several cable routes savings of several $100,000 €$ per year are possible. The application of the best cable diagnostic tool available today and result-based diagnostic knowledge are the fundamentals for finding weak cable sections which can be replaced individually, instead of replacing the entire routes [7].
XLPE cables were installed all around the world since the early 1980 s and already reach service life times of close to 40 years, whereas nobody can seriously define the life cycle of a XLPE cable.

How can the remaining life time of medium voltage power cables be estimated?

KEPCO Korea (Korean Electric Power Corporation) has addressed this question during the past years to their research group and found a unique solution and answer to this question. Korea Electric Power Corporation (KEPCO) can be considered as the leading pioneer who implemented cable diagnostic on a large scale for their entire distribution cable network. Since the implementation of VLF Tan Delta and VLF Partial Discharge Diagnostic in 2011, more than 15,000 cable routes were diagnosed in Korea, with the intention to understand the health condition of each individual cable. KEPCO's primary goal was always to strive for a position where a remaining lifetime of a XLPE cable can be estimated. With the vast amount of diagnostic data KEPCO developed a statistical tool that allows identifying a critical state of a cable when a cable failure is very likely to happen. Combining VLF Tan Delta (TD) and Partial Discharge (PD) diagnostic it is possible to identify and localize weak individual spots along a cable. After weak spots are cleared, the general aging condition of the cable can be judged by the new approach of KEPCO and the remaining life time can be estimated. This new tool is available to asset managers to help developing a preventive maintenance strategy for their power networks all around the world.

This study shall also reveal the applicability of the KEPCO approach to other distribution system operators (DSO). Diagnostic results are compared with the KEPCO approach in order to understand how XLPE

*Corresponding author: t.neier@baur.at 
cables of other utilities can be judged in a comparable way.

\section{Condition based asset management acc. Ti IEEE 400.2-2013 field guide}

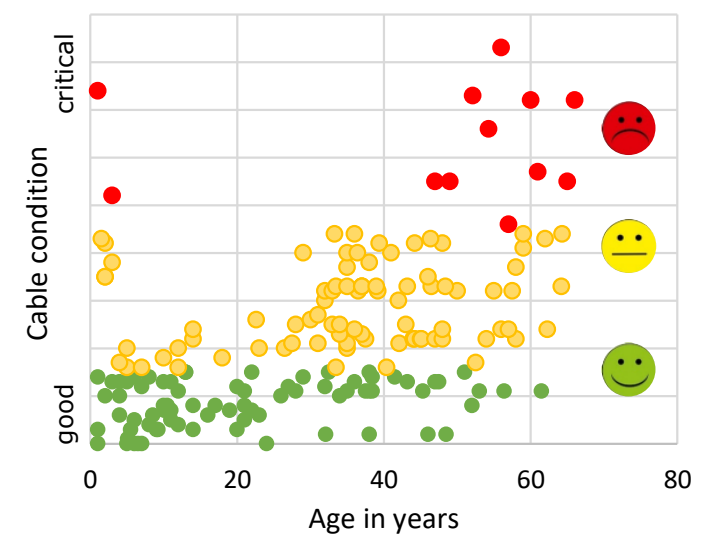

Fig. 1. Distribution of cable condition vs. duty period.

Categorizing underground cables based on the cable condition has become possible during the past years by application of VLF cable diagnostic techniques.

In 2013 IEEE 400.2 [1] established comprehensive evaluation criteria for classifying various cable types into different condition categories: "No action required", "Further study advised" and "Action required". The evaluation criterion is mainly based on VLF Tan Delta diagnostics. This enables the DSO to perform a condition based asset management strategy categorizing the cables depending on their condition (Figure 1).

Many Power utilities all around the world started the implementation of VLF TD diagnostic as a basis for the condition evaluation of MV cables. They often refer to the evaluation criteria provided by IEEE 400.2-2013 [1] which is proven to be useful for the cable health categorization.

IEEE 400.2-2013, [1, page 19] offers criteria based on a study in North America for aged cables. In detail, these are described in "Table 4 - Historical figures of merit for condition assessment of service-aged PE-based insulations (e.g. PE, XLPE, and TRXLPE) using 0.1 Hz" [1]. A graphical representation of IEEE 400.2 Table 4 is given in Figure 2.

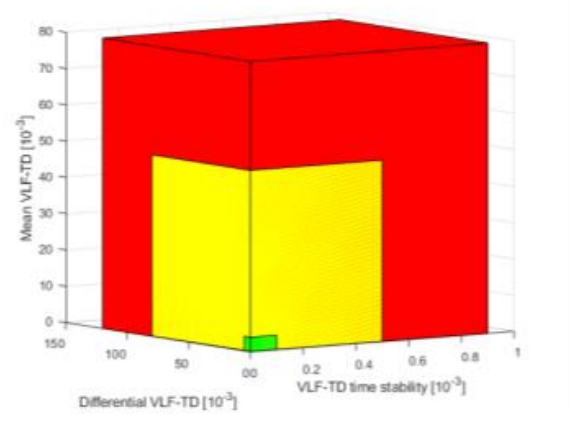

Fig. 2. 3D Illustration of IEEE 400.2-2013 evaluation logic, Table 4 .
The criteria in IEEE 400.2 table 4 are based on tan delta tests on a high number of cables in North America, where the overall distribution was declared by defining categories based on the pareto principle with $80 \% / 95 \%$ approach.

The application of these criteria lead to a wide range of cables being defined for conditions categorized as "no action required $(80 \%)$ and "further study advised" $(15 \%)$. The asset manager may have limited information on how to make decisions for relevant cable replacements.

These limitations need to be considered: For aged cable networks the large yellow range ("Further study advised") may lead to a unprecise categorization with the risk of an over- or underestimation of the real cable condition. Cables with singular weak spots turning into potential defects might not be identified and will not further indicate their probable severe condition. This may lead to unpredicted cable breakdowns.

Alternatively to these criteria, IEEE 400.2-2013 offers further evaluation criteria in the appendix that refer to early findings by German utilities in cooperation with the Technical University of Berlin. These criteria are available in appendix I, page 48, "Table I.1Alternate figures of merit for condition assessment of PE-based insulations (i.e., PE, XLPE)" [1], visualized in figure 3. In IEEE 400.2-2013 these criteria are mentioned as criteria applicable outside of North America.

Also these criteria are applied globally for new and aged cables. If these criteria are applied on aged cable networks, they are often categorized as "further study advised" or even "action required". These criteria can be referred to as very strict. As consequence only cables in excellent condition would be acceptable for service which is not feasible from a commercial point of view. Thus the applicability of these criteria for aged cables was questioned by KEPCO.

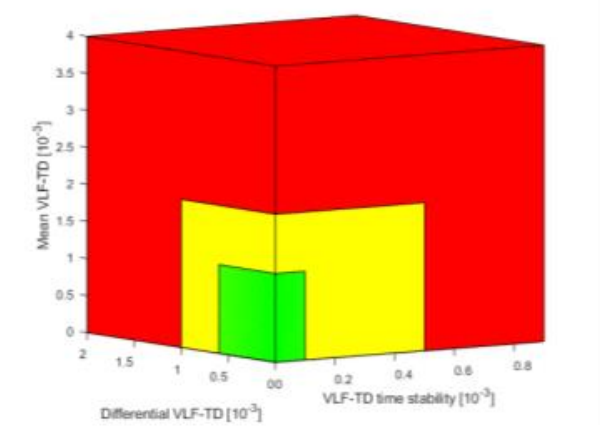

Fig. 3. 3D Illustration of IEEE 400.2-2013 evaluation logic, Table I.1 [1].

In figure 4 the two different evaluation criteria are compared based on identical scaling of the $\mathrm{x} / \mathrm{y} / \mathrm{z}-$ axis. It can be seen, that the criteria mentioned in table 4 of [1] (left graph) categorizes aged cables in a wide range, whereas the criteria mentioned in table I.1 of [1] (right graph) are very strict. Every condition exceeding the small range of green and yellow would be judged as "action required". 

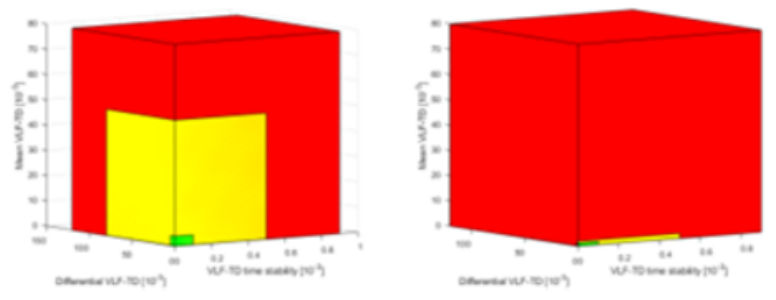

Fig. 4. 3D Illustration of IEEE 400.2-2013 evaluation criteria, comparison between Table 4 (left) and Table I.1 (right).

This motivates many DSOs around the world to find an alternative solution with more detailed condition estimation.

Already in 2011 KEPCO started the development of adapted evaluation criteria which are found to be more appropriate for the XLPE cable network in Korea. Details were published in [8].

\section{Combined cable diagnostic}

Cable joints with partial discharge (PD) activities and joints with water ingress need to be addressed differently than cables with healthy joints. In order to identify and localize these critical joints, KEPCO as well as many other utilities make use of the comprehensive diagnostic tools VLF Tan Delta (TD) with all its trend pattern details, Partial Discharge (PD) Diagnostic, Monitored Withstand Diagnostic [3]. With the help of Time Domain Reflectometry (TDR) [4] Singapore Power Grid identifies and localizes joints with water ingress by the use of TDR [2]. The smart combination of these methods allows localizing all weaknesses along a cable at a reduced voltage stress level (up to $1.5 \times U_{o}$ only, where $U_{o}$ is defined as phase to ground voltage) during VLF diagnostic. Most meaningful diagnostic results can be achieved and the cable condition can be evaluated. For aged medium voltage cables it is essential to keep the voltage stress during the diagnostic measurement as low as possible [3]. This low stress application differs significantly compared to a VLF Withstand Test. Here it shall be stated once again, that cable diagnostics can be used to evaluate the cable condition while avoiding any stressing voltage application. During a VLF Withstand Test as well as Monitored Withstand Test (MWT) much higher test voltages are applied with the aim of detecting singular weak spots by initiating a cable breakdown.

\section{Methodology of remaining life time estimation}

The methodology to address the question on the estimation of the Remaining Life Time (RLT) of cables was developed by KEPCO Korea analyzing their extensive VLF Tan Delta diagnostic field test results collected over many years. KEPCO identified that the key parameter of VLF Tan Delta is the time stability of the loss factor figures of Tan Delta. The stability of the Tan Delta figures combined with the trending direction has been defined as skirt value. In order to identify this grade of details, highest accuracy of the Tan Delta diagnostic device is required, providing accurate and stable Tan Delta values of at least $1 \times 10^{-5}$.

As an example, the graph in figure 5 shows a Tan Delta diagnostic diagram with Tan Delta values at three defined voltage levels comprising eight measurements per voltage level. The three phases and especially L1 shows an increasing trend characteristic indicating the presence of water tree aging. [6]

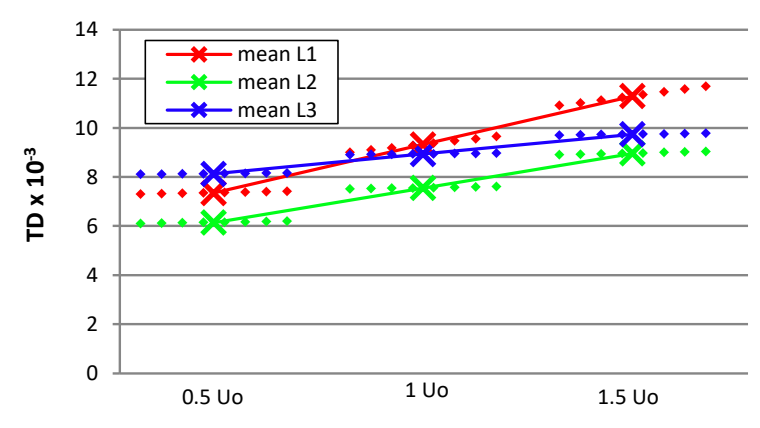

Fig. 5. Ref. 3536, Tan Delta pattern of water tree aged cable, three voltage levels with eight consecutive measurements.

As per KEPCOs publication [5] the complex Tan Delta pattern consists of Standard Deviation STD, Skirt value, Delta Tan Delta DTD as indication of Tan Delta versus voltage rise and Mean Tan Delta MTD. The Skirt value has a big advantage over the Standard Deviation. It allows describing the characteristics of various patterns of time-stability such as increasing, decreasing and oscillated very clearly. With this background, KEPCO has adopted the Skirt as well as the STDEV. These characteristics can be converted into a three dimensional vector where each cable is represented by its unique Rvalue:

$$
R=\sqrt{T D_{\text {norm }}^{2}+D T D_{\text {norm }}^{2}+\text { skirt }_{\text {norm }}^{2}}
$$

These R-values are shown as an extract of in total 45,000 Tan Delta diagnostic data points of $22.9 \mathrm{kV}$ XLPE cables in a three dimensional cluster (Figure 6). The right top corner is the area defined as economic limit, where cable failures are likely to occur and a reliable operation cannot be expected any longer. The yellow triangles indicate cables failures during operation.

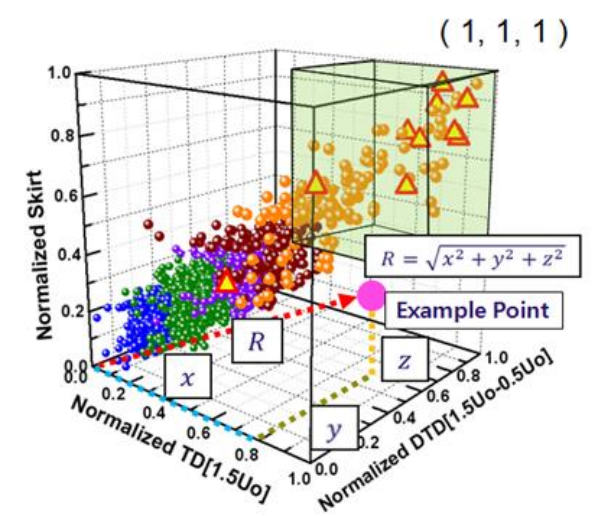

Fig. 6. 3D Graphic of $R$ values and cable failures [5]. 


\section{Estimation of remaining life time}

The statistical approach of estimating the remaining life time of cables is based on the statistics of 15,000 cable circuits measured, these are 45,000 cables providing 45,000 data points. This data set may be considered as the largest VLF Tan Delta data base of comparable cables worldwide. [5]

Figure 7 illustrates the cable life time assessment approach based on an example. KEPCO's statistical evaluation offered insight that cables below 13 years in service commonly do not show aging. Accordingly, a Degradation Starting Point (DSP) can be defined. This is the point, where aging is expected to start. The economical limit is set at a level where cables statistically failed or are expected to fail any moment. In order not to reach this level, a safety margin is determined by the DSO. This further defined level is defined as Critical Point $(C P)$.

In a simplified model the $y$-axis shall be scaled from 0 (new condition) to 10 units (maximum range of Tan Delta considered). Based on this assumption, an example of calculation can be presented. The statistical DSP of the cable network is 13 years. The limit of economical operation is 8 units. The DSO defined the critical cable condition point including a safety margin at 7 units. When reaching the Critical Point $(C P)$, the end of life time is considered.

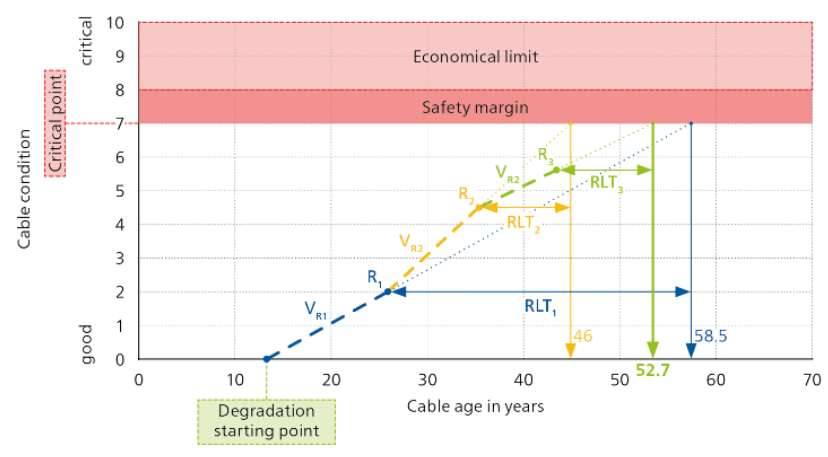

Fig. 7. Estimation of Remaining Life Time at different testing points.

Example: A cable tested after a duty period of 26 years is diagnosed at a level of aging condition $R_{l}$ scaling to 2.0 units. The aging speed can be calculated as $R_{l}$ over the aging period being the duty period minus the degradation starting point. Consequently, an average aging speed of 0.15 units per year can be calculated (2). The Remaining Life Time $(R L T)$ is calculated based on the statistical aging speed by extending the same aging speed $\left(V_{R}\right)$ until reaching the Critical Point (here $\left.C P_{l}=7\right)$. Following this, the Remaining Life Time $\left(R L T_{1}\right)$ with reference to the DSP would be 32.5 years (3) and reaching a total life time of 58.5 years.

$$
\begin{gathered}
V_{R_{1}}=\frac{R_{1}}{D P_{1}-D S P}=\frac{2}{26-13}=0.15 a^{-1} \\
R L T_{1}=\frac{C P-R_{1}}{V_{R 1}}=\frac{7-2}{0.15 a^{-1}}=32.5 a
\end{gathered}
$$

This calculation is based on the initial diagnostic measurement. It is advisable to repeat a diagnostic measurement after 5 to a maximum of 10 years. The implemented logic allows continuing and refining previous diagnostic results. In this example, the cable would be re-tested after 36 years in operation gaining an aging condition of $R_{2}$ of 4.5 . The precise and corrected aging speed $V_{R 2}$ with reference to the first measurement result $R_{l}$ indicates that the actual aging speed is either increased due to different load condition or the cable condition is weaker compared to the statistical average.

$$
\begin{gathered}
V_{R_{2}}=\frac{R_{2}-R_{1}}{D P_{2}-D P_{1}}=\frac{4.5-2}{36-26}=0.25 a^{-1} \\
R L T_{2}=\frac{C P-R_{2}}{V_{R 2}}=\frac{7-4.5}{0.25 a^{-1}}=10 a
\end{gathered}
$$

With an aging speed of 0.25 per year (4) the refined remaining life time is resulting to 10 years (5) and reaching a total life time of 46 years. This scenario could have the consequence of reduction of load in order to keep the aging lower and extend the life time. With reference to the result 2 , a re-test would be scheduled after further 8 years. The re-test after 44 years delivers a condition value of 5.7 and redefines the aging speed $V_{R 3}$ (6) indicating that the aging speed decreased as consequence of the lower load stress. The $R L T_{3}(7)$ is calculated to 8.7 years. Summing up, the cable will reach an estimated duty period of approximately 53 years.

$$
\begin{gathered}
V_{R_{3}}=\frac{R_{3}-R_{2}}{D P_{3}-D P_{2}}=\frac{5.7-4.5}{44-36}=0.15 a^{-1} \\
R L T_{3}=\frac{C P-R_{3}}{V_{R 3}}=\frac{7-5.7}{0.15 a^{-1}}=8.7 a
\end{gathered}
$$

Cable diagnostic data can be imported into the software and the result, shown in figure 8 will be shown with detailed information by phase, including the duty period, individual R-Index, degradation speed, remaining life time and date for next inspection. Further, the software compares the evaluation with respect to the relevant IEEE 400.2-2013 evaluation criteria.

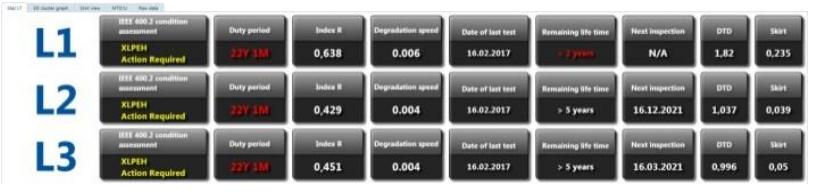

Fig. 8. Example of a cable where the remaining life time for L3 is estimated with less than 2 years.

\section{Results}

This life time estimation approach has been implemented by KEPCO for their power cable network in 2015. The cable diagnostic data collected until 2015 only comprised comparable $22.9 \mathrm{kV}$ XLPE cables of similar type, installation condition as well as joints. By 2015, the 
world's largest VLF Tan Delta database of 15,000 cable systems with approx. 5,000 circuit $\mathrm{km}$ and comparable test results was accumulated in the diagnostic database. In order to evaluate the logic of categorizing the cable condition and estimating the remaining life time of these circuits, a direct comparison with IEEE 400.2-2013 evaluation criteria (refer to figure 3 ) was done.

When using the evaluation criteria according to IEEE 400.2-2013 field guide, "Table 4 - Historical figures of merit for condition assessment of service-aged PE-based insulations (e.g., PE, XLPE, and TRXLPE) using $0.1 \mathrm{~Hz}$ " [1], out of the 15,000 cable systems, $255 \mathrm{~km}$ would be categorized as "action required". This is the highest category, where cable breakdowns have to be expected.

The evaluation of the same database using KEPCO's new life time estimation logic only judged $54 \mathrm{~km}$ as cables with remaining life time of less than two years ("action required"). This leads to the assumption that according to IEEE 400.2-2013 field guide criteria, $201 \mathrm{~km}$ would have been replaced unnecessarily even if they were not yet in a very critical condition. The study further revealed that the average life time in comparison to the IEEE 400.2-2013 field guide was increased by 11 years. Referring to the cost saving calculation also mentioned in the introduction section of this paper, this approach achieved the actual cost saving of 1.4 million USD in 2015 itself. The cost saving is based on avoiding $200 \mathrm{~km}$ unnecessary cable replacements immediately and offers the possibility to take advantage of increasing their life time to the naturally given limit.

\section{Verification of study}

For approval of this calculation a further evaluation was conducted. By 2015, approximately $11,000 \mathrm{~km}$ of $22.9 \mathrm{kV}$ cable were diagnosed. This represents approximately $25 \%$ of the entire MV network in Korea. Figure 9 shows the recorded failure statistics per year. The network failure rate of not yet diagnosed cables is rated at 3.37 failures per $1,000 \mathrm{~km}$ per year, which indicates a very healthy cable network. Cables with a duty period of less than 13 years indicate only 0.32 failures per $1,000 \mathrm{~km}$ per year. In reference to this excellent figure, cables that are judged with the KEPCO algorithm of estimating the remaining life time indicate a rate of 0.29 unexpected faults per $1,000 \mathrm{~km}$ per year only. The data shown in figure 9 reveals the excellent condition of KEPCO's medium voltage cable network where cable failures rarely happen. The estimation of the remaining life time allows to judge that cables can be operated for different duty periods in close alignment of their aging condition.

\begin{tabular}{|l|l|l|l|}
\hline & $\begin{array}{l}\text { New cable } \\
<13 \text { years }\end{array}$ & $\begin{array}{l}\text { Diagnosed } \\
\text { cables }\end{array}$ & $\begin{array}{l}\text { Not yet } \\
\text { diagnosed } \\
\text { cables }\end{array}$ \\
\hline $\begin{array}{l}\text { Failure rate } \\
\text { (fault/1.000 km) }\end{array}$ & 0,32 & 0,29 & 3,37 \\
\hline
\end{tabular}

Fig. 9. KEPCO failure rate per 1,000 km.
Power utilities aim for such a solution that allows scheduling maintenance and replacement work with respect to the real aging condition. If these figures are available for the entire cable network, a proactive preventive maintenance strategy can be established.

\section{Applicability for other power utilities}

Combined VLF Tan Delta and VLF Partial Discharge Diagnostics have been carried out in numerous power utilities all around the world during the past years and proved to be the most appropriate method to identify and localize weak spots in medium voltage distribution cables. Cable accessories are often subject to water ingress, tracking effects as well as weaknesses caused by partial discharge activities. All these weaknesses can be localized very well and replaced subsequently. After replacement of these faulty accessories the aged cable insulation usually defines the remain life time. The key question on the remaining life time of the aged cables is in the asset manager's mind and can hardly be answered by commonly used evaluation criteria. The introduced tool for estimation of the remaining life time, developed by KEPCO has drawn a lot attention already and was verified to be applicable on XLPE cable networks outside of KEPCO by other utilities.

Based on this interest, the correlation with a cable network of a power utility outside Korea compared with the KEPCO database has been investigated based on 240 cable diagnostic results on $11 \mathrm{kV}$ XLPE cables retrieved from a power utility in Asia.

The VLF TD diagnostic results of aged XLPE circuits without any unpredictable irregular joints as well as circuits with unpredictable accessories with water ingress, tracking effect and PD activities were taken into consideration. The cable circuits up to $1.500 \mathrm{~m}$ in length were selected. A simple upload of the VLF Tan Delta results with complementary entry of cable type and cable age is supported by the powerful software tool and saved in the database of the software.

The results were automatically evaluated based on KEPCO's algorithm and transferred to the $3 \mathrm{D}$ diagram forming a cluster (Figure 10).

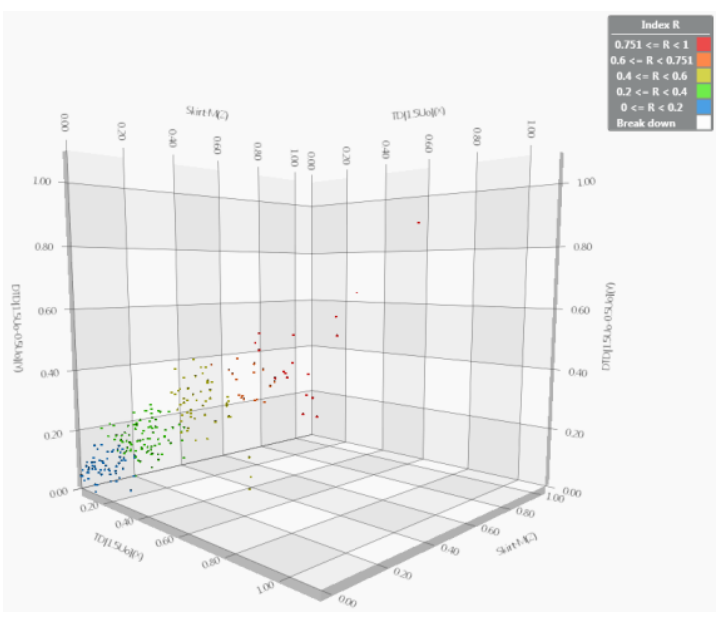

Fig. 10. 3D Data cluster of different DSO with 240 single cables. 
An impressive cluster was developed which shows very similar characteristics compared to the KEPCO cluster (Figure 11). Cables that included irregular joints with water ingress and/or PD activity were indicated in the critical range with red dots. Figure 11 indicates the reference data cluster of KEPCO with all scale values kept equal.

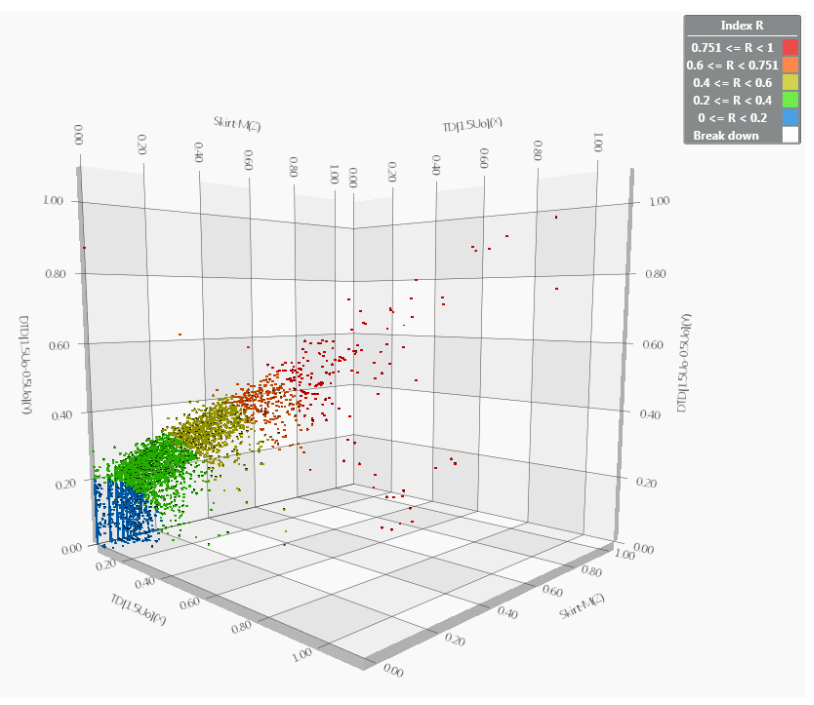

Fig. 11. 3D Reference data cluster by KEPCO with 45.000 cables.

A correlation can be seen with respect to the distribution of data points in the 3D diagram. The cluster position is very similar to the large KEPCO cluster. The application on a relatively small number of aged cables shows that several cables are in the critical range.

\section{Conclusion and outlook}

The study reveals that there is a great potential in cost saving for distribution system operators in case the cable life expectancy can be judged correctly. The statistical approach developed by KEPCO was possible, as the large amount of data can be grouped into a single characteristic group of homogeneous cables. The failure rate of diagnosed cable was significantly reduced to a minimum and proves the cable life time estimation approach, which was challenged on its applicability on diagnostic data retrieved from other power utilities MV network with excellent results. The correlation was proven by one particular utility. The distribution of the $\mathrm{R}$-indexes appeared to be in line with the reference data base. Therefore, the study allows the conclusion, that the evaluation algorithm developed for XLPE cables already used in Korea can be applied directly on XLPE cables for other utilities worldwide.

This understanding is in line with IEEE 400.2-2013 approach that defines that VLF TD evaluation criteria for XLPE cable of similar type can be applied commonly and with same criteria. It further supports the idea of IEEE that the criteria are equal or rather similar for different voltage levels of medium voltage networks.

The combination of VLF truesinus $\AA$ diagnostic measurements combined with this powerful tool delivers the answer for the estimation of remaining life time of medium voltage power cables and concludes for how long aged cables and cable sections can remain in service with high reliability.

The algorithm will be further studied to understand its applicability on cables used in several different regions around the world.

The authors gratefully acknowledge KEPCO Korea for sharing their knowledge and experience and allowing developing a tool that for the first time offers the possibility of life time estimation of medium voltage cables that can be used by power utilities all around the world. Further acknowledgment shall be given to the power utility who shared their valuable diagnostic data that was compared with the KEPCO database.

\section{References}

[1] IEEE400.2-2013, „Guide for Field Testing of Shielded Power Cable Systems Using Very Low Frequency," IEEE Standards Association, New York, USA, (2013)

[2] Dr. Cao et al, SP Power Grid Ltd., "Condition Monitoring of Distribution Cables using VLF Diagnostic Test with Tangent Delta, Time Domain Reflectometry and Partial Discharge Measurements," in CEPSI, Bangkok, (2016)

[3] M. Bawart, Austria, Endesa Distribution Electrica Spain, ,Improving Cable System Reliability with Monitored Withstand Diagnostics - featuring high efficiency at reduced test time," in Jicable 9th International Conference of Insulated Power Cables, Versailles, France, (2015)

[4] B. Neier, Cable diagnostic in $M V$ underground cable networks, 3.0 Hrsg., Sulz: BAUR, 102-110 (2015)

[5] Kim et al, IEEE, KEPCO, “A Study on Three Dimensional Assessment of the Aging Condition of Polymeric Medium Voltage Cables Applying VLF Tan Delta Diagnostic," IEEE Transaction on Dielectrics and Electrical Insulation, (2014)

[6] Neier, „Combined Application of Diagnostics Tools for MV Underground Cables" in Jicable, Versailles, (2015)

[7] M. Jenny, BAUR Austria, ,VLF Sinus $0.1 \mathrm{~Hz}$ universal voltage source for testing and diagnosis of medium voltage power cables" in Cigre, Paris, France, (2014)

[8] L. Kim, K. Korea, "VLF Tan-Delta Criteria for $X L P E$ Insulated Power Cables in KEPCO" in Jicable, Paris, France" (2011) 